Milica Nikodijevic $\square$

Zivojin Stamenkovic

Jelena Petrovic

Milos Kocic

https://doi.org/10.21278/TOF.444014420

ISSN 1333-1124

eISSN 1849-1391

\title{
UNSTEADY FLUID FLOW AND HEAT TRANSFER THROUGH A POROUS MEDIUM IN A HORIZONTAL CHANNEL WITH AN INCLINED MAGNETIC FIELD
}

\begin{abstract}
Summary
This paper investigates the unsteady flow and heat transfer of a viscous, incompressible, and electrically conducting fluid through a porous medium in a horizontal channel. The basic physical properties of the fluid and the porous medium are constant. The fluids considered are those with the Prandtl number less than 1. The channel walls are made of horizontal permeable plates, which are at constant but different temperatures. Fluid suction/injection through the plates occurs at a velocity perpendicular to the plates, whose intensity is a cosine function of time. The applied external magnetic field is homogeneous and inclined in relation to the transverse plane of the channel. The problem is dealt with through an inductionless approximation. Fluid flow is instigated by constant pressure drops along the channel. The equations used to describe the problem are transformed to dimensionless forms and solved analytically using the perturbation method. Approximate analytical expressions for dimensionless fluid flow velocity and dimensionless temperature are determined as functions of the following physical parameters: Prandtl number, Hartmann number, porosity factor, frequency, amplitude, and magnetic field inclination angle. Numerical results are presented as diagrams and tables and are used to analyse the influence of physical parameters on the fluid flow velocity and temperature.
\end{abstract}

Key words: $\quad$ Fluid flow, Heat transfer, Prandtl number, Hartmann number, Perturbation method

\section{Introduction}

The publication of Darcy's [1] paper, in which he experimentally determined that the water flow velocity through sand is proportional to the pressure gradient, initiated and practically enabled subsequent theoretical research into the fluid flow through porous media. A number of researchers began to investigate this subject area, the main reason being that fluid flows frequently occur in nature, industry, and technological processes. Fluid flows are found in astrophysics, geophysics, metalworking, processing industry, chemical industry, pharmaceutical industry, storage engineering, petroleum industry, hydrology, medicine, physiology, biology, and in many other fields. Their noteworthy application in medicine 
M. Nikodijevic, Z. Stamenkovic, J. Petrovic, M. Kocic
Unsteady Fluid Flow and Heat Transfer Through a Porous Medium in a Horizontal Channel with an Inclined Magnetic Field

includes the study of blood flow through human and animal blood vessels, urine transport from the kidneys to the bladder, food ingestion through the oesophagus, movement through the gastro-intestinal tract, flow through the lungs, and gallstones passing from the gall bladder through the bile duct. More recent areas of study include the flow of liquid metals, electrolytes, and ionized gases. These are only some of the instances of fluid flow through a porous medium. Since the development of magnetohydrodynamics and the publication of Shercliff's [2] book, numerous researchers have been studying magnetohydrodynamic (MHD) fluid flows. Umavathi et al. [3] studied the unsteady MHD flow of two non-mixing fluids in a horizontal channel. Using the perturbation method, they obtained approximate analytical expressions for fluid flow velocities and temperatures as functions of physical parameters. The results were presented in figures and tables and were analysed. Mosayebidorcec et al. [4] investigated the influence of variable fluid properties, the Hartmann number, the Hall current, the Reynolds number, the suction velocity on the unsteady MHD Couette flow, and the heat transfer of a dusty fluid. Umavathi et al. [5] studied the oscillatory flow and heat transfer through a composite porous medium in a horizontal channel. The energy equation takes the viscous and Darcy dissipation into consideration. The authors obtained approximate analytical solutions for fluid velocity, which they presented graphically and also analysed the influence of physical parameters. Petrović et al. [6] studied the steady flow of two non-mixing fluids through a porous medium between two horizontal plates at constant but different temperatures. The external magnetic field was homogeneous and inclined in the direction of the flow, whereas the external electric field was homogeneous and perpendicular to the vertical plane of channel symmetry. The authors analysed the obtained analytical solutions for fluid velocity and temperature. Gupta and Jain [7] analysed the unsteady MHD flow through a porous medium between two horizontal plates, where the top plate is permeable with exponential suction and constant velocity along its plane, while the bottom plate stretches and is perpendicularly influenced by a homogeneous magnetic field. To solve the problemdescribing equations in their dimensionless form, the authors used the perturbation method. They examined the effects of physical parameters on the velocity and shear stress. Kumar and Agarwal [8] investigated the MHD pulsatile flow through a porous medium of a fluid sandwiched between viscous fluids inside permeable horizontal plates. They used the Beavers-Joseph boundary conditions for sliding on the horizontal plates. The formulated dimensionless equations were solved using the perturbation method and the influence of physical parameters on the shear velocity and stress was analysed. Krishna and Reddy [9] studied the unsteady MHD convective flow of second grade fluid through a porous medium in a rotating parallel plate channel. They obtained analytical solutions using the Laplace transform. Lakshmanna and Venkateswarlu [10] investigated the same problem as Krishna and Reddy using only a slightly different approach.

Numerous studies deal with the mass and the heat transfer around a vertical plate and in a vertical channel through a porous medium and under the influence of a magnetic field. Chamkha [11] investigated the unsteady MHD convective heat and mass transfer past a semiinfinite vertical permeable moving plate through a porous medium and under the influence of a homogenous transverse magnetic field. Equations were solved using the perturbation method. Ahmed et al. [12] examined the unsteady MHD free convective flow past a vertical porous plate immersed in a porous medium. They also considered the Hall current, thermal diffusion, and the heat source/sink. Kumar et al. [13] studied the unsteady MHD periodic flow and heat transfer of viscous fluid through a porous vertical channel under the influence of a homogenous transverse magnetic field. They also used the perturbation model to solve the formulated equations. Manna et al. [14] investigated the effects of radiation on unsteady MHD free convective flow past an oscillating vertical porous plate embedded in a porous medium. Chand et al. [15] examined the influence of the Hall effect on the radiating and 
chemically reacting MHD oscillatory convective flow in a rotating porous vertical channel. It was assumed that the fluid flow was caused by the pressure gradient as a periodic function of time. Choudhury and Das [16] studied the unsteady visco-elastic MHD convective flow and heat transfer through porous media with a vertical porous plate in the presence of radiation and a chemical reaction. The dimensionless equations were solved using the perturbation method. Uwanta and Usman [17] investigated the influence of Soret and Dufour effects on the unsteady MHD free convective heat and mass transfer flow through a porous medium over a vertical channel. They considered viscous dissipation and constant suction, while the thermal conductivity coefficient was a linear function of temperature. Barik et al. [18] examined the unsteady free convective MHD flow and the mass transfer through a porous medium with a heat source and a chemical reaction between vertical porous plates in a rotating system. The formulated equations were solved using the perturbation method and the solutions were subsequently analysed. Prakash et al. [19] studied the effects of thermal radiation, buoyancy force, and magnetic field on oscillatory dusty fluid flow in a vertical channel filled with a saturated porous medium. Kupala and Reddy [20] investigated the unsteady MHD convective heat and mass transfer of a Casson fluid past a semi-infinite vertical plate with a heat source/sink. The plate is immersed in a porous medium. Kumar [21] examined the MHD peristaltic transportation of a conducting blood flow with a porous medium through an inclined coaxial vertical channel. The author obtained analytical expressions for axial velocity, pressure gradient, and shear stress and analysed them. Using the Mathematica software, Misra and Adhirary [22] studied the heat and the mass transfer in the MHD oscillatory blood flow through porous arterioles in the presence of a chemical reaction. Falade et al. [23] investigated the effects of suction/injection and slide velocity along the channel wall on the unsteady MHD oscillatory flow through a vertical channel saturated with a porous medium. Krishna et al. [24] examined the influence of radiation and Hall effects on the unsteady MHD oscillatory convective flow of second grade fluid through a porous medium in a vertical channel. The fluid moved due to the oscillatory pressure gradient, while the magnetic field was homogeneous and inclined in relation to the channel. The temperature of one of the walls changed periodically.

This paper analyses the unsteady flow and the heat transfer of an incompressible and electrically conducting fluid through a porous medium in a horizontal channel. The channel walls consist of horizontal permeable plates, which are kept at constant but different temperatures. The applied external magnetic field is homogeneous and inclined in relation to the transverse plane of the channel. It is assumed that the value of the Reynolds magnetic number is low; thus, the induced magnetic field can be disregarded. Fluid movement is caused by a constant pressure gradient along the channel.

\section{Formulation of the problem}

The present study considers the unsteady flow and the heat transfer of a viscous, incompressible, and electrically conducting fluid through a porous medium between two fixed horizontal plates which are at a constant distance $h$. The top plate is at a constant temperature $T_{w 1}$, and the bottom at a constant temperature $T_{\mathrm{w} 2}\left(T_{\mathrm{w} 1}>T_{\mathrm{w} 2}\right)$. The applied external magnetic field is homogeneous and inclined at an angle $\alpha$ in relation to the transverse plane of the channel, while the intensity of its induction is $B$. It is assumed that the value of the Reynolds magnetic number is low; thus, the induced magnetic field can be disregarded with respect to the applied magnetic field, i.e. the problem can be considered in an inductionless approximation. The flow is caused by a constant pressure gradient along the $x$-axis. A Cartesian coordinate system is introduced (Fig. 1) such that the $x$-axis goes along the channel and corresponds to the bottom plate, while the $y$-axis is perpendicular to the plates. 
M. Nikodijevic, Z. Stamenkovic, J. Petrovic, M. Kocic
Unsteady Fluid Flow and Heat Transfer Through a Porous Medium in a Horizontal Channel with an Inclined Magnetic Field

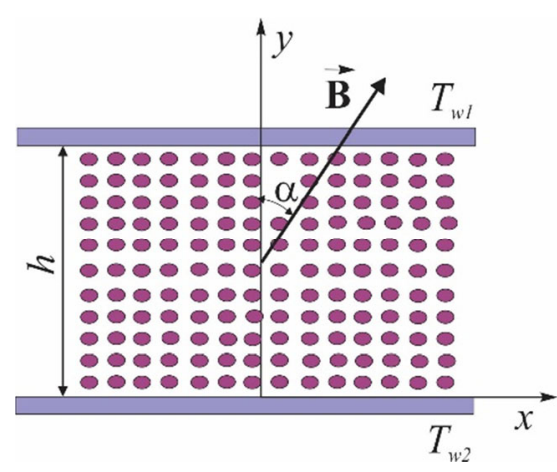

Fig. 1 Physical configuration

Since the plates are infinite, all physical quantities except pressure depend solely on the $\mathrm{y}$ coordinate and the time $t$. Starting from the continuity equation, the Navier-Stokes equation expanded by the inclusion of the Lorentz force, which arises due to the movement of electrically conductive fluid in the magnetic field and due to the porous medium resistance force, and the energy equation, which includes Joule heating and energy dissipation caused by a porous medium, it follows that the described problem can be expressed using the following equations:

$$
\begin{aligned}
& \frac{\partial v}{\partial y}=0, \\
& \frac{\partial u}{\partial t}+v \frac{\partial u}{\partial y}=-\frac{1}{\rho} \frac{\partial p}{\partial x}+\frac{\mu}{\rho} \frac{\partial^{2} u}{\partial y^{2}}-\left(\frac{\mu}{\rho K}+\lambda^{2} \frac{\sigma B^{2}}{\rho}\right) u, \\
& \frac{\partial T}{\partial t}+v \frac{\partial T}{\partial y}=\frac{k}{\rho c_{p}} \frac{\partial^{2} T}{\partial y^{2}}+\frac{\mu}{\rho c_{p}}\left(\frac{\partial u}{\partial y}\right)^{2}+\frac{1}{c_{p}}\left(\frac{\mu}{\rho K}+\lambda^{2} \frac{\sigma B^{2}}{\rho}\right) u^{2},
\end{aligned}
$$

and boundary conditions:

$$
u(0)=0, u(h)=0, T(0)=T_{w 2}, T(h)=T_{w 1},
$$

where: $u, v$-longitudinal and transversal fluid velocity, respectively; $p$ - pressure; $\rho, \mu, k, \sigma$ and $c_{p}$-density, dynamic viscosity, thermal conductivity, electrical conductivity, and specific heat at constant pressure, respectively; $T$-fluid temperature; $K$-porous medium permeability; and $\lambda=\cos \alpha$. It should be noted that this is a simplified mathematical model, but quite appropriate, as it includes the influences of the medium porosity, magnetic field and its inclination, and transversal velocity. The projection of the expanded Navier - Stokes equation onto the $y$-axis has been omitted because pressure distribution is not considered in this study. It follows from Eq.(1) that $v$ does not depend on $y$; instead, the velocity is defined as a random cosine function of time $t$. The transversal velocity $v$ can be assumed to take the following form:

$$
v=v_{0}[1+\varepsilon A \cos (\omega t)],
$$

where: $A$-real constant; $\omega$-frequency; and $\varepsilon$-small quantity such that $\varepsilon A \leq 1$. It is assumed that the velocity changes periodically as a function of time around the mean value 
$v_{0}$, which is not equal to zero. For $\varepsilon A=0$, the transversal fluid velocity is constant. By introducing the dimensionless quantities

$$
\begin{aligned}
& y^{*}=\frac{y}{h}, t^{*}=\frac{\mu}{\rho h^{2}} t, u^{*}=\frac{u}{U_{0}}, \omega^{*}=\frac{\omega}{\omega_{0}}, v^{*}=\frac{v}{v_{0}}, \\
& \theta=\frac{T-T_{w 2}}{T_{w 1}-T_{w 2}}, U_{0}=\frac{h^{2} P}{\mu}, P=-\frac{\partial p}{\partial x}, \omega_{0}=\frac{\mu}{\rho h^{2}}, v_{0}=\frac{\mu}{\rho h},
\end{aligned}
$$

equations (2) and (3) are transformed into the following dimensionless forms:

$$
\begin{aligned}
& \frac{\partial u}{\partial t}+v \frac{\partial u}{\partial y}=\frac{\partial^{2} u}{\partial y^{2}}-R u+1, \\
& \frac{\partial \theta}{\partial t}+v \frac{\partial \theta}{\partial y}=\frac{1}{\operatorname{Pr}} \frac{\partial^{2} \theta}{\partial y^{2}}+E c\left(\frac{\partial u}{\partial y}\right)^{2}+E c R u^{2},
\end{aligned}
$$

and boundary conditions (4) into the corresponding dimensionless boundary conditions:

$$
u(0)=0, u(1)=0, \theta(0)=0, \theta(1)=1,
$$

where the following notation is introduced, for brevity purposes:

$$
\Lambda=\frac{h^{2}}{K}, R=\Lambda+\lambda^{2} H a^{2}, E c=\frac{U_{0}^{2}}{c_{p}\left(T_{w 1}-T_{w 2}\right)}, H a=B h \sqrt{\frac{\sigma}{\mu}}, \operatorname{Pr}=\frac{\mu c_{p}}{k} .
$$

It must be noted that the symbol * has been omitted, to simplify the notation, but it will be implied that the quantities are dimensionless; the newly-introduced symbols, which will henceforth be used but are not commented here, are appended.

\section{Solution}

By assuming the solution in the forms

$$
\begin{aligned}
& u(y, t)=u_{0}(y)+\varepsilon \cos (\omega t) u_{1}(y)+O\left(\varepsilon^{2}\right)+\ldots \\
& \theta(y, t)=\theta_{0}(y)+\varepsilon \cos (\omega t) \theta_{1}(y)+O\left(\varepsilon^{2}\right)+\ldots
\end{aligned}
$$

and by using them to substitute parts of Eq. (7) and Eq. (8), while disregarding small quantities of a higher order than $\varepsilon^{2}$ and separating non-harmonic and harmonic expressions, the following equation pairs with corresponding boundary conditions are obtained:

$$
\begin{aligned}
& \frac{\mathrm{d} u_{0}}{\mathrm{~d} y}=\frac{\mathrm{d}^{2} u_{0}}{\mathrm{~d} y^{2}}-R u_{0}+1, \\
& \frac{\mathrm{d} \theta_{0}}{\mathrm{~d} y}=\frac{1}{\operatorname{Pr}} \frac{\mathrm{d}^{2} \theta_{0}}{\mathrm{~d} y^{2}}+E c\left(\frac{\mathrm{d} u_{0}}{\mathrm{~d} y}\right)^{2}+E c R u_{0}^{2}, \\
& u_{0}(0)=0, u_{0}(1)=0, \theta_{0}(0)=0, \theta_{0}(1)=1
\end{aligned}
$$


M. Nikodijevic, Z. Stamenkovic, J. Petrovic, M. Kocic
Unsteady Fluid Flow and Heat Transfer Through a Porous Medium in a Horizontal Channel with an Inclined Magnetic Field

and

$$
\begin{aligned}
& \frac{\mathrm{d}^{2} u_{1}}{\mathrm{~d} y^{2}}-\frac{\mathrm{d} u_{1}}{\mathrm{~d} y}+R_{1} u_{1}=A \frac{\mathrm{d} u_{0}}{\mathrm{~d} y} \\
& \frac{1}{P r} \frac{\mathrm{d}^{2} \theta_{1}}{\mathrm{~d} y^{2}}-\frac{\mathrm{d} \theta_{1}}{\mathrm{~d} y}+\Omega \theta_{1}=A \frac{\mathrm{d} \theta_{0}}{\mathrm{~d} y}-2 E c R u_{0} u_{1}-2 E c \frac{\mathrm{d} u_{0}}{\mathrm{~d} y} \frac{\mathrm{d} u_{1}}{\mathrm{~d} y}, \\
& u_{1}(0)=0, u_{1}(1)=0, \theta_{1}(0)=0, \theta_{1}(1)=0
\end{aligned}
$$

where the following notation is introduced, for brevity purposes:

$$
\Omega=\omega \tan (\omega t), R_{1}=\Omega-R .
$$

The solution to Eq. (13) is given by the expression

$$
u_{0}(y)=C_{1} \exp \left(r_{1} y\right)+C_{2} \exp \left(r_{2} y\right)+\frac{1}{R}
$$

where the constants of integration $C_{1}$ and $C_{2}$ and the quantities $r_{1}$ and $r_{2}$ are given by the following expressions:

$$
C_{1}=\frac{1}{R} \frac{\exp \left(r_{2}\right)-1}{\exp \left(r_{1}\right)-\exp \left(r_{2}\right)}, C_{2}=\frac{1}{R} \frac{1-\exp \left(r_{1}\right)}{\exp \left(r_{1}\right)-\exp \left(r_{2}\right)}, r_{1,2}=\frac{1}{2}(1 \pm \sqrt{1+4 R}) .
$$

The problem of Prandtl number values being less than one is discussed below. This is typical of liquid metals, such as mercury $(\operatorname{Pr} \approx 0.021)$, liquid sodium $(\operatorname{Pr} \approx 0.005)$, and certain solutions, e.g. metal-ammonia solutions $(P r \approx 0.7)$ (Scheel and Schumacher, [25]).

When $\operatorname{Pr}$ number values are less than one, the solution to Eq. (14) is given by the expression

$$
\begin{aligned}
& \theta_{0}(y)=C_{3}+C_{4} \exp (P r y)+A_{1} \exp \left(2 r_{1} y\right)+A_{2} \exp \left(2 r_{2} y\right)+ \\
& A_{3} \exp (y)+A_{4} \exp \left(r_{1} y\right)+A_{5} \exp \left(r_{2} y\right)+A_{6} y,
\end{aligned}
$$

where the constants of integration $C_{3}$ and $C_{4}$ and the notation introduced for brevity purposes are given by the expressions

$$
\begin{aligned}
& C_{3}=\frac{R_{3}-1-R_{2} \exp (\operatorname{Pr})}{\exp (\operatorname{Pr})-1}, C_{4}=\frac{1+R_{2}-R_{3}}{\exp (\operatorname{Pr})-1}, A_{1}=\frac{\operatorname{PrEc}\left(R+r_{1}^{2}\right) C_{1}^{2}}{2 r_{1}\left(\operatorname{Pr}-2 r_{1}\right)}, \\
& A_{2}=\frac{\operatorname{PrEc}\left(R+r_{2}^{2}\right) C_{2}^{2}}{2 \mathrm{r}_{2}\left(\operatorname{Pr}-2 r_{2}\right)}, A_{3}=\frac{R_{12}\left(r_{1} r_{2}+R\right) C_{1} C_{2}}{\operatorname{Pr}-1}, A_{4}=\frac{R_{12} C_{1}}{r_{1}\left(\operatorname{Pr}-r_{1}\right)}, \\
& A_{5}=\frac{R_{12} C_{2}}{r_{2}\left(\operatorname{Pr}-r_{2}\right)}, R_{2}=A_{1}+A_{2}+A_{3}+A_{4}+A_{5}, A_{6}=\frac{E c}{R}, R_{12}=2 \operatorname{PrEc}, \\
& R_{3}=A_{1} \exp \left(2 r_{1}\right)+A_{2} \exp \left(2 r_{2}\right)+A_{3} \exp (1)+A_{4} \exp \left(r_{1}\right)+A_{5} \exp \left(r_{2}\right)+A_{6} .
\end{aligned}
$$


Equations (14) and (17) with boundary conditions (18) are solved next. After the substitution of $u_{0}(y)$, i.e. expression (20), in Eq. (16), it follows that its solution depends on the quantity

$$
D=1+4\left(\Lambda+\lambda^{2} H a^{2}-\Omega\right)
$$

For $D>0$, the solution is given by the expression

$$
u_{1}^{(1)}(y)=C_{5} \exp \left(n_{1} y\right)+C_{6} \exp \left(n_{2} y\right)+u_{1 P}(y)
$$

where

$$
u_{1 P}(y)=A_{7} \exp \left(r_{1} y\right)+A_{8} \exp \left(r_{2} y\right)
$$

while the constants of integration $C_{5}$ and $C_{6}$ and the notation introduced for brevity purposes are given by the expressions

$$
\begin{aligned}
& C_{5}=\frac{R_{4} \exp \left(n_{2}\right)-R_{5}}{\exp \left(n_{1}\right)-\exp \left(n_{2}\right)}, C_{6}=\frac{R_{5}-R_{4} \exp \left(n_{1}\right)}{\exp \left(n_{1}\right)-\exp \left(n_{2}\right)}, n_{1,2}=\frac{1}{2}\left(1 \pm \sqrt{1-4 R_{1}}\right) \\
& R_{5}=A_{7} \exp \left(r_{1}\right)+A_{8} \exp \left(r_{2}\right), R_{4}=A_{7}+A_{8}, A_{7}=\frac{A r_{1} C_{1}}{r_{1}^{2}-r_{1}+R_{1}}, A_{8}=\frac{A r_{2} C_{2}}{r_{2}^{2}-r_{2}+R_{1}} .
\end{aligned}
$$

For $D=0$, the solution to Eq. (16) is:

$$
u_{1}^{(2)}(y)=\left(C_{7}+C_{8} y\right) \exp (y / 2)+u_{1 P}(y)
$$

where the constants of integration $C_{7}$ and $C_{8}$ are given by the expressions

$$
C_{7}=-R_{4}, C_{8}=R_{4}-R_{5} \exp (-1 / 2)
$$

while the solution for $D<0$ is

$$
R u_{1}^{(3)}(y)=C_{9} \cos \left(R_{6} y\right) \exp (y / 2)+C_{10} \sin \left(R_{6} y\right) \exp (y / 2)+u_{1 P}(y)
$$

and the constants of integration are given by the expressions

$$
C_{9}=-R_{4}, C_{10}=\frac{R_{4} \cos \left(R_{6}\right)-R_{5} \exp (-1 / 2)}{\sin \left(R_{6}\right)}, R_{6}=\frac{1}{2} \sqrt{|D|} .
$$

It is now possible to formulate a solution to Eq. (17), which is found to depend on quantity $D_{1}$, given by the expression

$$
D_{1}=\operatorname{Pr}(\operatorname{Pr}-4 \Omega) \text {. }
$$

For $D_{1}>0$, the solution is unique and is given by the expression

$$
\theta_{1}(y)=C_{11} \exp \left(m_{1} y\right)+C_{12} \exp \left(m_{2} y\right)+\theta_{p}(y)
$$

where

$$
\begin{aligned}
& \theta_{p}(y)=\theta_{p}^{*}(y)+A_{15} \exp \left(\left(r_{1}+n_{1}\right) y\right)+A_{16} \exp \left(\left(r_{1}+n_{2}\right) y\right)+ \\
& A_{17} \exp \left(\left(r_{2}+n_{1}\right) y\right)+A_{18} \exp \left(\left(r_{2}+n_{2}\right) y\right)+A_{19} \exp \left(n_{1} y\right)+A_{20} \exp \left(n_{2} y\right)
\end{aligned}
$$


M. Nikodijevic, Z. Stamenkovic, J. Petrovic, M. Kocic
Unsteady Fluid Flow and Heat Transfer Through a Porous Medium in a Horizontal Channel with an Inclined Magnetic Field

and

$$
\begin{aligned}
& \theta_{p}^{*}(y)=A_{9} \exp (\text { Pry })+A_{10} \exp \left(2 r_{1} y\right)+A_{11} \exp \left(2 r_{2} y\right)+ \\
& A_{12} \exp (y)+A_{13} \exp \left(r_{1} y\right)+A_{14} \exp \left(r_{2} y\right)+A_{21}
\end{aligned}
$$

which, in addition to the condition $D_{1}>0$, also satisfies the condition $D>0$. The constants of integration $C_{11}$ and $C_{12}$ and the notation introduced for brevity purposes are given by the following expressions:

$$
\begin{aligned}
& C_{11}=\frac{R_{7} \exp \left(m_{2}\right)-R_{8}}{\exp \left(m_{1}\right)-\exp \left(m_{2}\right)}, C_{12}=-C_{11}-R_{7}, m_{1,2}=\frac{1}{2}\left(\operatorname{Pr} \pm \sqrt{D_{1}}\right), \\
& A_{10}=\frac{2 \operatorname{Pr}\left[A r_{1} A_{1}-E c\left(R+r_{1}^{2}\right) C_{1} A_{7}\right]}{2 r_{1}\left(2 r_{1}-\operatorname{Pr}\right)+S}, A_{11}=\frac{2 \operatorname{Pr}\left[A r_{2} A_{2}-E c\left(R+r_{2}^{2}\right) C_{2} A_{8}\right]}{2 r_{2}\left(2 r_{2}-\operatorname{Pr}\right)+S}, \\
& A_{12}=\frac{\operatorname{Pr}\left[A A_{3}-2 E c\left(R+r_{1} r_{2}\right)\left(C_{1} A_{8}+C_{2} A_{7}\right)\right]}{1-\operatorname{Pr}+S}, A_{13}=\frac{\operatorname{Pr}\left(A r_{1} A_{4}-2 E c A_{7}\right)}{r_{1}\left(r_{1}-\operatorname{Pr}\right)+S}, \\
& A_{14}=\frac{\operatorname{Pr}\left(A r_{2} A_{5}-2 E c A_{8}\right)}{r_{2}\left(r_{2}-\operatorname{Pr}\right)+S}, A_{15}=-\frac{R_{12}\left(R+r_{1} n_{1}\right) C_{1} C_{5}}{\left(r_{1}+n_{1}\right)\left(r_{1}+n_{1}-\operatorname{Pr}\right)+S}, R_{7}=\sum_{i=9}^{21} A_{i}, \\
& A_{16}=-\frac{R_{12}\left(R+r_{1} n_{2}\right) C_{1} C_{6}}{\left(r_{1}+n_{2}\right)\left(r_{1}+n_{2}-\operatorname{Pr}\right)+S}, A_{17}=-\frac{R_{12}\left(r_{2} n_{1}\right) C_{2} C_{5}}{\left(r_{2}+n_{1}\right)\left(r_{2}+n_{1}-\operatorname{Pr}\right)+S}, \\
& A_{18}=-\frac{R_{12}\left(R+r_{2} n_{2}\right) C_{2} C_{6}}{\left(r_{2}+n_{2}\right)\left(r_{2}+n_{2}-\operatorname{Pr}\right)+S}, A_{19}=-\frac{R_{1}}{n_{1}\left(n_{1}-\operatorname{Pr}\right)+S}, A_{21}=\frac{A A_{6}}{\Omega}, \\
& A_{20}=-\frac{R_{12} C_{6}}{n_{2}\left(n_{2}-\operatorname{Pr}\right)+S}, S=\operatorname{Pr} \Omega, A_{9}=\frac{\operatorname{Pr} A C_{4}}{\Omega}, R_{8}^{*}=A_{9} \exp (\operatorname{Pr})+ \\
& A_{10} \exp \left(2 r_{1}\right)+A_{11} \exp \left(2 r_{2}\right)+A_{12} \exp (1)+A_{13} \exp \left(r_{1}\right)+A_{14} \exp \left(r_{2}\right)+A_{21}, \\
& R_{8}=R_{8}^{*}+A_{15} \exp \left(r_{1}+n_{1}\right)+A_{16} \exp \left(r_{1}+n_{2}\right)+A_{17} \exp \left(r_{2}+n_{1}\right)+ \\
& A_{18} \exp \left(r_{2}+n_{2}\right)+A_{19} \exp \left(n_{1}\right)+A_{20} \exp \left(n_{2}\right) .
\end{aligned}
$$

For $D_{1}=0$, the solution is also unique and is given by the expression below:

$$
\theta_{1}(y)=\left(C_{13}+C_{14} y\right) \exp (\operatorname{Pr} y / 2)+\theta_{p}(y)
$$

which simultaneously satisfies the condition $D>0$. The constants of integration $C_{13}$ and $C_{14}$ are given by the expressions

$$
C_{13}=-R_{7}, C_{14}=\mathrm{R}_{7}-\mathrm{R}_{8} \exp (-\operatorname{Pr} / 2) \text {. }
$$

It must be noted that solutions for $D_{1} \geq 0$ exist only when $D>0$.

If $D_{1}<0$, there are three solutions for $D>0$, which are given by the following expression:

$$
\theta_{1}(y)=C_{15} \cos \left(R_{9} y\right) \exp (\text { Pry } / 2)+C_{16} \sin \left(R_{9} y\right) \exp (\text { Pry } / 2)+\theta_{p}(y),
$$


where the constants of integration $C_{15}$ and $C_{16}$ are given by the expressions

$$
C_{15}=-R_{7}, C_{16}=\frac{R_{7} \cos \left(R_{9}\right)-R_{8} \exp (-\operatorname{Pr} / 2)}{\sin \left(R_{9}\right)}, R_{9}=\frac{1}{2} \sqrt{\left|D_{1}\right|} .
$$

For $D<0$ :

$$
\begin{aligned}
& \theta_{1}(y)=\left[C_{17} \cos \left(R_{9} y\right)+C_{18} \sin \left(R_{9} y\right)\right] \exp (\operatorname{Pr} y / 2)+\theta_{p}^{*}(y)- \\
& {\left[A_{22} \cos \left(R_{6} y\right)+A_{23} \sin \left(R_{6} y\right)\right] \exp \left(\alpha_{1} y\right)-} \\
& {\left[A_{24} \cos \left(R_{6} y\right)+A_{25} \sin \left(R_{6} y\right)\right] \exp \left(\alpha_{2} y\right)-} \\
& {\left[A_{26} \cos \left(R_{6} y\right)+A_{27} \sin \left(R_{6} y\right)\right] \exp (y / 2),}
\end{aligned}
$$

where the constants of integration $C_{17}$ and $C_{18}$ are given by the expressions

$$
C_{17}=-R_{15}, C_{18}=\frac{R_{15} \cos \left(R_{9}\right)-R_{16} \exp (-\operatorname{Pr} / 2)}{\sin \left(R_{9}\right)},
$$

which utilize the following notation:

$$
\begin{aligned}
& R_{10}=\operatorname{Pr} A, R_{11}=2 \operatorname{PrEcR}, R_{13}=C_{9} / 2+C_{10} R_{6}, R_{14}=C_{10} / 2-C_{9} R_{6}, \\
& B_{1}=\left(R_{11} C_{9}+r_{1} R_{12} R_{13}\right) C_{1}, B_{2}=\left(R_{11} C_{10}+r_{1} R_{12} R_{14}\right) C_{1}, \\
& B_{3}=\left(R_{11} C_{9}+r_{2} R_{12} R_{13}\right) C_{2}, B_{4}=\left(R_{11} C_{10}+r_{2} R_{12} R_{14}\right) C_{2}, \\
& B_{5}=R_{12} C_{9}, B_{6}=R_{12} C_{10}, \alpha_{1}=r_{1}+1 / 2, \alpha_{2}=r_{2}+1 / 2, \\
& S_{1}=\alpha_{1}\left(\alpha_{1}-\operatorname{Pr}\right)-R_{6}^{2}+S, S_{2}=R_{6}\left(2 \alpha_{1}-\operatorname{Pr}\right), A_{22}=\frac{B_{1} S_{1}-B_{2} S_{2}}{S_{1}^{2}+S_{2}^{2}}, \\
& A_{23}=\frac{B_{1} S_{2}+B_{2} S_{1}}{S_{1}^{2}+S_{2}^{2}}, A_{24}=\frac{B_{3} S_{3}-B_{4} S_{4}}{S_{3}^{2}+S_{4}^{2}}, A_{25}=\frac{B_{3} S_{4}+B_{4} S_{3}}{S_{3}^{2}+S_{4}^{2}}, A_{26}=\frac{B_{5} S_{5}-B_{6} S_{6}}{S_{5}^{2}+S_{6}{ }^{2}}, \\
& A_{27}=\frac{B_{5} S_{6}+B_{6} S_{5}}{S_{5}^{2}+S_{6}^{2}}, S_{3}=\alpha_{2}\left(\alpha_{2}-\operatorname{Pr}\right)-R_{6}^{2}+S, S_{4}=R_{6}\left(2 \alpha_{2}-\operatorname{Pr}\right), \\
& S_{5}=1 / 4-\operatorname{Pr} / 2-R_{6}^{2}+S, S_{6}=R_{6}(1-\operatorname{Pr}), \\
& R_{15}=\sum_{i=9}^{14} A_{i}+A_{21}-A_{22}-A_{24}-A_{26}, \\
& R_{16}=R_{8}^{*}-\left[A_{22} \cos \left(R_{6}\right)+A_{23} \sin \left(R_{6}\right)\right] \exp \left(\alpha_{1}\right)- \\
& {\left[A_{24} \cos \left(R_{6}\right)+A_{25} \sin \left(R_{6}\right)\right] \exp \left(\alpha_{2}\right)-\left[A_{26} \cos \left(R_{6}\right)+A_{27} \sin \left(R_{6}\right)\right] \exp (1 / 2)}
\end{aligned}
$$

and for $D=0$ :

$$
\begin{aligned}
& \theta_{1}(y)=C_{19} \cos \left(R_{9} y\right) \exp (\operatorname{Pry} / 2)+C_{20} \sin \left(R_{9} y\right) \exp (\text { Pry } / 2)+ \\
& \theta_{p}^{*}(y)-A_{28} \exp \left(\alpha_{1} y\right)-A_{29} y \exp \left(\alpha_{1} y\right)-A_{30} \exp \left(\alpha_{2} y\right)- \\
& A_{31} y \exp \left(\alpha_{2} y\right)-A_{32} \exp (y / 2)-A_{33} y \exp (y / 2)
\end{aligned}
$$

The constants of integration and the notation used are given by the following expressions: 


$$
\begin{aligned}
& C_{19}=-R_{20}, C_{20}=\frac{R_{20} \cos \left(R_{9}\right)-R_{1} \exp (-\operatorname{Pr} / 2)}{\sin \left(R_{9}\right)}, B_{7}=R_{11} C_{1} C_{7}+\left(C_{7} / 2+C_{8}\right) r_{1} R_{12} C_{1}, \\
& B_{8}=\left(R_{11}+r_{1} R_{12} / 2\right) C_{1} C_{8}, B_{9}=R_{11} C_{2} C_{7}+\left(C_{7} / 2+C_{8}\right) r_{2} R_{12} C_{2}, \\
& B_{10}=\left(R_{11}+r_{2} R_{12} / 2\right) C_{2} C_{8}, B_{11}=R_{12} C_{7}, B_{12}=R_{12} C_{8}, R_{17}=\alpha_{1}\left(\alpha_{1}-\operatorname{Pr}\right)+S, \\
& R_{18}=\alpha_{2}\left(\alpha_{2}-\operatorname{Pr}\right)+S, R_{19}=1 / 4-\operatorname{Pr} / 2+S, A_{28}=\frac{B_{7}}{R_{17}}+\frac{\operatorname{Pr}-2 r_{1}-1}{R_{17}{ }^{2}} B_{8} \\
& A_{30}=\frac{B_{9}}{R_{18}}+\frac{\operatorname{Pr}-2 r_{2}-1}{R_{18}{ }^{2}} B_{10}, A_{31}=\frac{B_{10}}{R_{18}}, A_{32}=\frac{B_{11}}{R_{19}}+\frac{\operatorname{Pr}-1}{R_{19}{ }^{2}} B_{12}, A_{33}=\frac{B_{12}}{R_{19}} \\
& R_{20}=\sum_{i=9}^{14} A_{i}+A_{21}-A_{28}-A_{30}-A_{32}, R_{21}=R_{8}^{*}-\left(A_{28}+A_{29}\right) \exp \left(\alpha_{1}\right)- \\
& \left(A_{30}+A_{31}\right) \exp \left(\alpha_{2}\right)-\left(A_{32}+A_{33}\right) \exp (1 / 2), A_{29}=\frac{B_{8}}{R_{17}} .
\end{aligned}
$$

Thus, we have formulated the approximate analytical expressions for the dimensionless fluid flow velocity in the channel and the dimensionless fluid temperature in the channel.

\section{Result analysis}

For better readability and a more efficient analysis, a portion of the obtained results is shown in figures and tables. Figure 2 shows the profiles of longitudinal dimensionless velocity for different values of the Hartmann number and for different physical quantities. It is noticeable that the increase in the Hartmann number value, i.e. in the intensity of the applied external magnetic field, causes the longitudinal velocity reduction in the channel and the flattening of its profile.

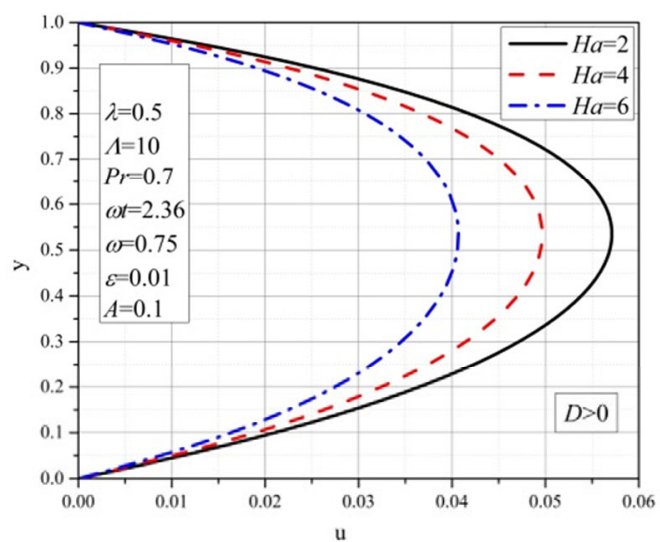

Fig. 2 Dimensionless velocity profiles for different Hartmann number $(\mathrm{Ha})$ values

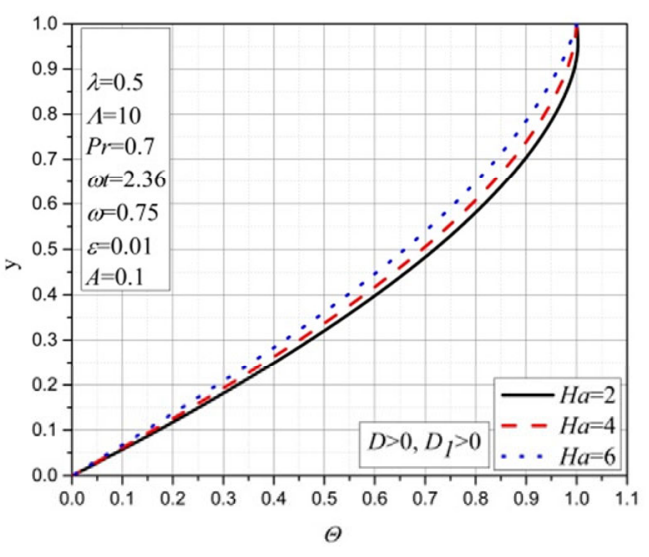

Fig. 3 Dimensionless temperature profiles for different Hartmann number $(\mathrm{Ha})$ values

For each value of the Hartmann number, the velocity reaches its maximum intensity just above the half of the channel height. When the intensity of the applied external magnetic field increases, the flow and the friction on the channel walls become reduced.

This is due to the facts that the increase in external magnetic field intensity increases the Lorentz force intensity and that the pressure gradient in the direction of the primary flow remains constant. These conclusions are also valid in case the values of physical quantities are such that the discriminant is $D<0$. 
Figure 3 shows the profiles of dimensionless fluid temperature in the channel for different values of the Hartmann number and for different physical quantities, whose values are identical to those for velocity profiles in Figure 2. It is noticeable that the increase in the Hartmann number causes the fluid temperature reduction in the channel. For the value $H a=2$, the heat is transferred from the fluid to the top channel wall, whereas for $H a$ values of 4 and 6, the heat is transferred from the top channel wall to the fluid. These conclusions also apply when the values of physical quantities are such that the discriminants are $D<0$ and $D_{1}<0$.

Tables 1 and 2 provide numerical values for the distribution of dimensionless longitudinal velocity, i.e. primary flow velocity, and of dimensionless temperature of the fluid, respectively, in the cases when $D>0$ and $D_{1}>0$. Table 1 shows that the primary flow velocity of the fluid increases up to the $55 \%$ of the channel height, and then decreases as the $\varepsilon A$ factor increases. The dimensionless temperature of the fluid increases together with the $\varepsilon A$ factor over the entire cross-section of the channel, as shown in Table 2.

Figure 4 shows the profiles of velocity for different values of the porosity factor $\Lambda$. It is noticeable that the higher values of this factor lead to decreased intensity of the velocity and the flattening of the profile, thus reducing the flow in the channel. The most intensive increase in velocity occurs near the channel walls for the lowest value of the porosity factor; this value is also associated with the highest friction on the walls. Such effects are caused by a resistance force that occurs due to the existence of solid phase in the porous medium. These conclusions are also valid in case the values of physical quantities are such that the discriminant is $D<0$.

Table 1 Dimensionless longitudinal velocity for different values of factor $\varepsilon A$

\begin{tabular}{|c|c|c|c|c|c|c|c|}
\hline \multirow{2}{*}{$y$} & \multicolumn{3}{|c|}{$u$} & \multirow{2}{*}{$y$} & \multicolumn{3}{|c|}{$u$} \\
\cline { 8 - 9 } \cline { 7 - 8 } & $\varepsilon A=0$ & $\varepsilon A=0.4$ & $\varepsilon A=0.8$ & & $\varepsilon A=0$ & $\varepsilon A=0.4$ & $\varepsilon A=0.8$ \\
\hline 1 & 0 & 0 & 0 & 0.45 & 0.05595 & 0.05636 & 0.05677 \\
\hline 0.95 & 0.01425 & 0.01385 & 0.01345 & 0.4 & 0.05397 & 0.05453 & 0.0551 \\
\hline 0.9 & 0.02574 & 0.02512 & 0.02451 & 0.35 & 0.05109 & 0.05179 & 0.05248 \\
\hline 0.85 & 0.03492 & 0.03422 & 0.03353 & 0.3 & 0.04729 & 0.04807 & 0.04886 \\
\hline 0.8 & 0.04214 & 0.04147 & 0.04079 & 0.25 & 0.0425 & 0.04333 & 0.04417 \\
\hline 0.75 & 0.04771 & 0.04712 & 0.04653 & 0.2 & 0.03664 & 0.03747 & 0.03831 \\
\hline 0.7 & 0.05183 & 0.05138 & 0.05092 & 0.15 & 0.0296 & 0.03037 & 0.03114 \\
\hline 0.65 & 0.05471 & 0.05442 & 0.05412 & 0.1 & 0.02127 & 0.02189 & 0.02251 \\
\hline 0.6 & 0.05648 & 0.05636 & 0.05624 & 0.05 & 0.01146 & 0.01183 & 0.01221 \\
\hline 0.55 & 0.05723 & 0.05729 & 0.05736 & 0 & 0 & 0 & 0 \\
\hline 0.5 & 0.05704 & 0.05728 & 0.05753 & & & & \\
\hline
\end{tabular}

Table 2 Dimensionless temperature for different values of factor $\varepsilon A$

\begin{tabular}{|c|c|c|c|c|c|c|c|}
\hline \multirow{2}{*}{$y$} & \multicolumn{3}{|c|}{$\theta$} & \multirow{2}{*}{$y$} & \multicolumn{3}{|c|}{$\theta$} \\
\cline { 2 - 5 } \cline { 6 - 8 } & $\varepsilon A=0$ & $\varepsilon A=0.4$ & $\varepsilon A=0.8$ & & $\varepsilon A=0$ & $\varepsilon A=0.4$ & $\varepsilon A=0.8$ \\
\hline 1 & 1 & 1 & 1 & 0.45 & 0.45157 & 0.45176 & 0.45195 \\
\hline 0.95 & 0.95036 & 0.95039 & 0.95042 & 0.4 & 0.40151 & 0.4017 & 0.40189 \\
\hline 0.9 & 0.90064 & 0.9007 & 0.90076 & 0.35 & 0.35142 & 0.3516 & 0.35178 \\
\hline 0.85 & 0.85088 & 0.85096 & 0.85105 & 0.3 & 0.30131 & 0.30147 & 0.30164 \\
\hline
\end{tabular}


M. Nikodijevic, Z. Stamenkovic, J. Petrovic, M. Kocic
Unsteady Fluid Flow and Heat Transfer Through a Porous Medium in a Horizontal Channel with an Inclined Magnetic Field

\begin{tabular}{|c|c|c|c|c|c|c|c|}
0.8 & 0.80107 & 0.80118 & 0.8013 & 0.25 & 0.25116 & 0.25132 & 0.25147 \\
\hline 0.75 & 0.75124 & 0.75137 & 0.7515 & 0.2 & 0.20099 & 0.20112 & 0.20126 \\
\hline 0.7 & 0.70137 & 0.70152 & 0.70167 & 0.15 & 0.15079 & 0.1509 & 0.15101 \\
\hline 0.65 & 0.65147 & 0.65164 & 0.65181 & 0.1 & 0.10057 & 0.10065 & 0.10072 \\
\hline 0.6 & 0.60155 & 0.60172 & 0.6019 & 0.05 & 0.05031 & 0.05035 & 0.05039 \\
\hline 0.55 & 0.55159 & 0.55177 & 0.55196 & 0 & 0 & 0 & 0 \\
\hline 0.5 & 0.50159 & 0.50178 & 0.50197 & & & & \\
\cline { 1 - 6 }
\end{tabular}

Figure 5 shows the profiles of dimensionless temperature in the channel for different values of the porosity factor $\Lambda$ and for different physical quantities, whose values are identical to those for velocity profiles in Figure 4. For the values of physical quantities, the discriminants are $D>0$ and $D_{1}>0$. It is noticeable that higher values of the porosity factor, i.e. lower permeability of porous media, can be associated with lower fluid temperatures in the channel. For the values $\Lambda=5$ and $\Lambda=10$, heat is transferred from the fluid to the top wall and vice versa for the value $\Lambda=15$. These conclusions also apply when the values of physical quantities are such that the discriminants are $D<0$ and $D_{1}<0$.

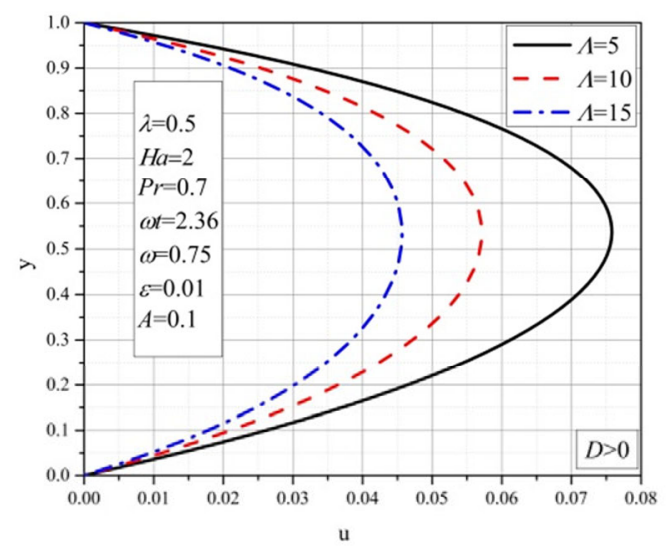

Fig. 4 Dimensionless velocity profiles for different values of porosity factor $\Lambda$

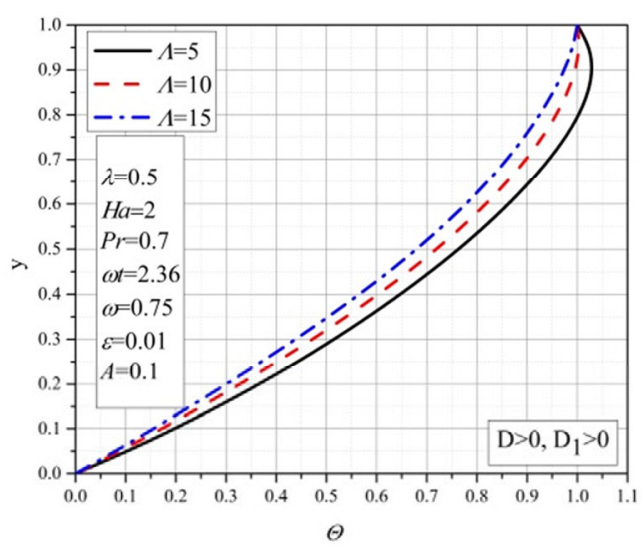

Fig. 5 Dimensionless temperature profiles for different values of porosity factor $\Lambda$

Figure 6 shows the profiles of dimensionless velocity for different values of factor $\lambda$ and for the values of physical quantities given in the figure for which the discriminant value is $D<0$. It is noticeable that the increase in factor $\lambda$, i.e. increase in the angle of the external magnetic field to the direction of the primary flow, leads to decreased velocity and flow of the fluid and reduced friction on the channel walls. For each value of factor $\lambda$, the velocity reaches its maximum intensity just above the half of the channel height. This is due to the fact that the increased factor $\lambda$ increases the Lorentz force intensity in the direction of the primary flow. These conclusions are also valid in case the values of physical quantities are such that the discriminant is $D>0$.

Figure 7 shows the profiles of dimensionless temperature in the channel for different values of factor $\lambda$ and for different physical quantities, whose values are identical to those for velocity profiles in Figure 6. For the values of physical quantities, the discriminants $D$ and $D_{1}$ are less than zero. It is noticeable that higher values of factor $\lambda$ can be associated with lower fluid temperatures in the channel. For the values $\lambda=0.5$ and $\lambda=0.705$, heat is transferred from the fluid to the top wall and vice versa for the value $\lambda=0.865$. 


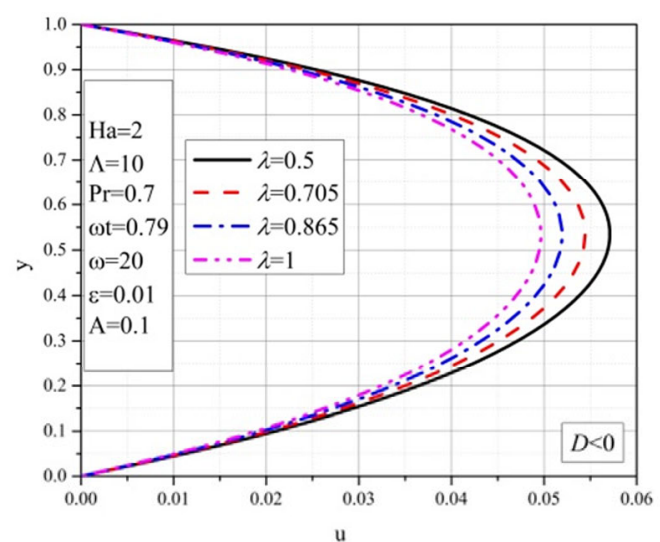

Fig. 6 Dimensionless velocity profiles for different values of factor $\lambda$

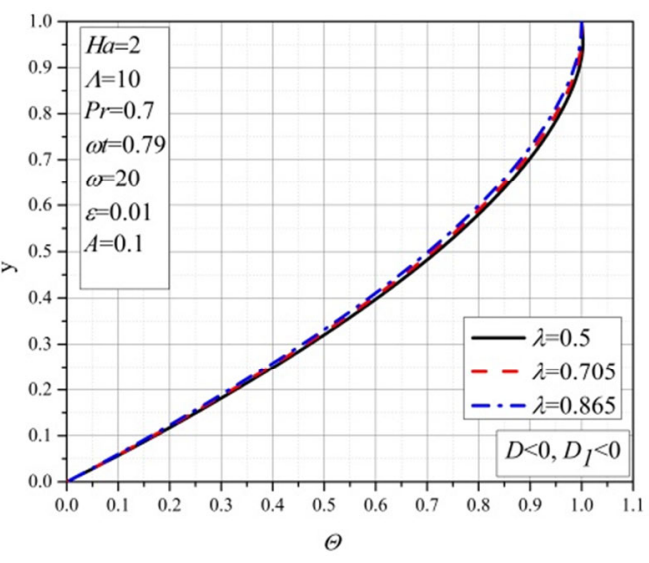

Fig. 7 Dimensionless temperature profiles for different values of factor $\lambda$

These conclusions also apply when the values of physical quantities are such that the discriminants $D$ and $D_{1}$ are greater than zero.

Tables 3 and 4 provide numerical values for the distribution of dimensionless longitudinal velocity and dimensionless temperature of the fluid for different values of the frequency factor $\omega t$, respectively, in the cases when $D>0$ and $D_{1}>0$. The tables show that both the velocity and the temperature decrease as the $\omega t$ factor increases, whereby the decrease is more prominent in the bottom half of the channel.

Table 3 Dimensionless longitudinal velocity for different values of factor $\omega t$

\begin{tabular}{|c|c|c|c|c|c|c|c|}
\hline & \multicolumn{3}{|c|}{$u$} & & \multicolumn{3}{c|}{$u$} \\
\hline$y$ & $\omega t=1.571$ & $\omega t=2.357$ & $\omega t=3.142$ & $y$ & $\omega t=1.571$ & $\omega t=2.357$ & $\omega t=3.142$ \\
\hline 1 & 0 & 0 & 0 & 0.45 & 0.055947 & 0.055948 & 0.055949 \\
\hline 0.95 & 0.014247 & 0.014246 & 0.014246 & 0.4 & 0.053969 & 0.053971 & 0.053971 \\
\hline 0.9 & 0.025739 & 0.025737 & 0.025736 & 0.35 & 0.051095 & 0.051096 & 0.051097 \\
\hline 0.85 & 0.034917 & 0.034916 & 0.034915 & 0.3 & 0.04729 & 0.047292 & 0.047293 \\
\hline 0.8 & 0.042143 & 0.042141 & 0.042141 & 0.25 & 0.042498 & 0.0425 & 0.042501 \\
\hline 0.75 & 0.047705 & 0.047704 & 0.047703 & 0.2 & 0.036638 & 0.03664 & 0.036641 \\
\hline 0.7 & 0.051835 & 0.051834 & 0.051833 & 0.15 & 0.029604 & 0.029606 & 0.029607 \\
\hline 0.65 & 0.054712 & 0.054712 & 0.054711 & 0.1 & 0.021266 & 0.021268 & 0.021268 \\
\hline 0.6 & 0.056476 & 0.056476 & 0.056476 & 0.05 & 0.011462 & 0.011463 & 0.011463 \\
\hline 0.55 & 0.057229 & 0.057229 & 0.057229 & 0.00 & $-1.2 \mathrm{E}-24$ & 0 & 0 \\
\hline 0.5 & 0.057039 & 0.057039 & 0.05704 & & & & \\
\cline { 1 - 3 }
\end{tabular}

Table 4 Dimensionless temperature for different values of factor $\omega t$

\begin{tabular}{|c|c|c|c|c|c|c|c|}
\hline & \multicolumn{3}{|c|}{$\theta$} & & \multicolumn{3}{c|}{$\theta$} \\
\hline$y$ & $\omega t=1.571$ & $\omega t=2.357$ & $\omega t=3.142$ & $y$ & $\omega t=1.571$ & $\omega t=2.357$ & $\omega t=3.142$ \\
\hline 1 & 1 & 1 & 1 & 0.45 & 0.451568 & 0.451568 & 0.451567 \\
\hline 0.95 & 0.950358 & 0.950359 & 0.950358 & 0.4 & 0.401511 & 0.401511 & 0.40151 \\
\hline 0.9 & 0.900643 & 0.900643 & 0.900643 & 0.35 & 0.351423 & 0.351423 & 0.351423 \\
\hline 0.85 & 0.850878 & 0.850878 & 0.850878 & 0.3 & 0.301307 & 0.301307 & 0.301306 \\
\hline
\end{tabular}


M. Nikodijevic, Z. Stamenkovic, J. Petrovic, M. Kocic
Unsteady Fluid Flow and Heat Transfer Through a Porous Medium in a Horizontal Channel with an Inclined Magnetic Field

\begin{tabular}{|c|c|c|c|c|c|c|c|}
0.8 & 0.801075 & 0.801075 & 0.801074 & 0.25 & 0.251163 & 0.251163 & 0.251162 \\
\hline 0.75 & 0.751239 & 0.751239 & 0.751238 & 0.2 & 0.200992 & 0.200992 & 0.200992 \\
\hline 0.7 & 0.701372 & 0.701372 & 0.701372 & 0.15 & 0.150795 & 0.150795 & 0.150795 \\
\hline 0.65 & 0.651475 & 0.651475 & 0.651475 & 0.1 & 0.100569 & 0.100569 & 0.100568 \\
\hline 0.6 & 0.601547 & 0.601547 & 0.601546 & 0.05 & 0.050307 & 0.050307 & 0.050307 \\
\hline 0.55 & 0.551586 & 0.551586 & 0.551586 & 0.00 & $1.05 \mathrm{E}-13$ & $1.06 \mathrm{E}-13$ & $1.05 \mathrm{E}-13$ \\
\hline 0.5 & 0.501593 & 0.501593 & 0.501593 & \multicolumn{1}{|c}{} \\
\cline { 1 - 7 }
\end{tabular}

Figure 8 shows the profiles of dimensionless fluid temperature for different values of the Prandtl number and for different physical quantities, as given in the figure. It is noticeable that the higher values of the Prandtl number can be associated with higher dimensionless temperatures in the channel and that for the value of $P r=0.7$, heat is transferred from the fluid to the top wall and vice versa for the values $\operatorname{Pr}=0.21$ and $\operatorname{Pr}=0.005$. For the lower Prandtl value, heat is usually transferred by means of conduction. These conclusions also apply when the values of physical quantities are such that the discriminants $D$ and $D_{1}$ are greater than zero.

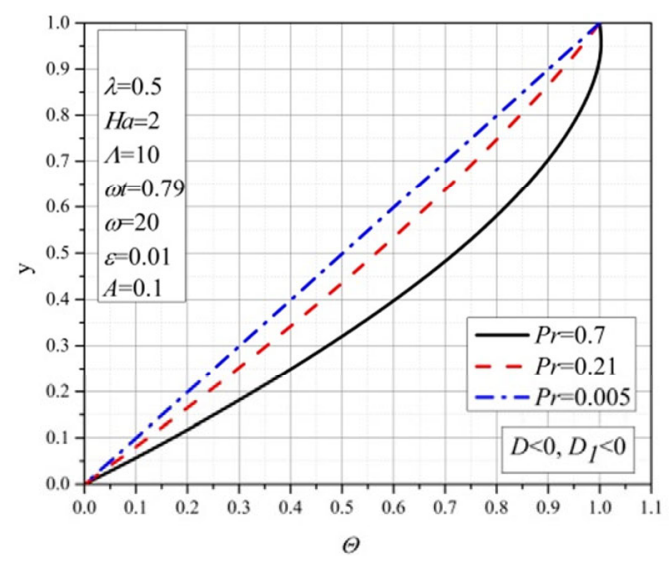

Fig. 8 Dimensionless velocity profiles for different Prandtl number $(\mathrm{Pr})$ values

\section{Conclusion}

This paper analysed the unsteady MHD flow and heat transfer through a porous medium in a horizontal channel with permeable walls. For the sake of generality, an arbitrary magnetic field inclination in relation to the primary fluid flow direction was selected, while the physical properties of the fluid and the porous medium were constant. Solutions to the equations in dimensionless form were obtained using the perturbation method, after which the approximate analytical expressions for dimensionless primary flow velocity and dimensionless temperature of the fluid were determined. Numerical values of the obtained analytical expressions were presented in diagrams and tables for different values of flow parameters and porous medium factors.

The analysis of the results led to several conclusions about the impact of the analysed quantities on fluid flow and heat transfer. It was observed that the combination of fluids with different physical properties, porous medium, amplitude of secondary the flow velocity oscillation, and external field intensity and its inclination can be used to control the fluid flow and heat transfer in the channel. It is possible to reduce the dimensionless velocity of primary fluid flow and the dimensionless temperature by increasing the Hartmann number values, 
which is achieved, for instance, by increasing the intensity of magnetic induction of the applied external magnetic field, by increasing the inclination of the field in relation to the direction of primary fluid flow, or by increasing the medium porosity factor. The dimensionless temperature of the fluid can also be reduced with lower Prandtl number values. In addition, proper selection of physical parameters can create conditions where the amplitude of secondary flow velocity oscillation will increase/decrease the dimensionless temperature in the channel cross-section and decrease the velocity across the entire or half of the crosssection. This can be significant for practical technical application.

\section{REFERENCES}

[1] Darcy, H. (1856). Fontainespubliques de la ville de Dijon. Librairie des Corps Imperiaux des Pontset Chausses et des Mines.

[2] Shercliff, J.A. (1965). A Text Book of Magnetohydrodynamics. London: Pergamon Press.

[3] Umavathi, J. C., A. J. Chamkha, A. Mateen and J. P. Kumar (2008). Unsteady magneto-hydrodynamic two fluid flow and heat transfer in a horizontal channel. Heat and Technology Vol. 26 (2), pp.121 - 133. https://doi.org/10.1007/s00231-004-0565-x

[4] Mosayebidorcheh, S., M. Hatami, D. D. Ganji, T. Mosayebidorcheh and S. M. Mirmohammadsadeghi (2015). Investigation of Transient MHD Couette Flow and Heat Transfer of Dusty Fluid with Temperature - Dependent Properties. Journal of Applied Fluid Mechanics Vol. 8(4), pp. 921 - 929. https://doi.org/10.18869/acadpub.jafm.67.223.23949

[5] Umavathi, J. C., A. J. Chamkha, A. Mateen and A. Al - Mudhaf (2009). Unsteady Oscillatory Flow and Heat Transfer in a Horizontal Composite Porous Medium Channel. Nonlinear Analysis: Modelling and Control Vol. 14(3), pp. 397 - 415. https://doi.org/10.15388/na.2009.14.3.14503

[6] Petrovic, J. D., Ž. M. Stamenkovic, M. M. Kocic and M. D. Nikodijevic (2016). Porous medium magnetohydrodynamic flow and heat transfer of two immisible fluids. Thermal Science Vol. 20(5), pp. 1405 - 1417. https://doi.org/10.2298/tsci16s5405p

[7] Gupta, V. G and A. Jain (2016). Back flow analysis of unsteady MHD fluid flow over stretching surface with Hall effect in presence of permeability. International Journal of Advances in Applied Mathematics and Mechanics Vol. 3(4), pp. $102-113$.

[8] Kumar, D. and M. Agarwal (2018). MHD Pulsatile Flow of a Conducting Fluid Sandwiched Between Viscous Fluids Inside Permeable Beds. Konnralp Journal of Mathematics Vol. 6(1), pp. 63 - 75.

[9] Krishna, M. V. and G. S. Reddy (2016). Unsteady MHD convective flow of Second grade fluid through a porous medium in a Rotating parallel plate channel with temperature dependent source. IOP Conf. Series: Materials Science and Engineering 149(012216). https://doi.org/10.1088/1757-899x/149/1/012216

[10] Lakshmanna B. and S. Venkateswarlu (2018). Heat transfer on MHD Rotating non - Newtonian Fluid Flow through Parallel Plate Porous Channel. International Journal of Applied Engineering Research Vol. 13(10), pp. $8200-8204$.

[11] Chamkha, A. J. (2004). Unsteady MHD convective heat and mass transfer past a semi-infinite vertical permeable moving plate with heat absorption. International Journal of Engineering Science 42, pp. 217 230. https://doi.org/10.1016/s0020-7225(03)00285-4

[12] AAhmed, N., H. Kalitan and D. P. Barua (2010). Unsteady MHD free convective flow past a vertical porous plate immersed in a porous medium with Hall current, thermal diffusion and heat source. International journal of Engineering, Science and Technology, Vol. 2(6), pp. 59 - 74. https://doi.org/10.4314/ijest.v2i6.63699

[13] Kumar, A., C. L. Varshney and S. Lab (2010). Perturbation technique to unsteady MHD periodic flow of viscous fluid through a planar channel. Journal of Engineering and Technology Research Vol. 2(4), pp. $73-81$.

[14] Manna, S. S., S. Das and R. N. Jana (2012). Effects of radiation on unsteady MHD free convective flow past an oscillating vertical porous plate embedded in a porous medium with oscillatory heat flux. Advances in Applied Science Research Vol. 3(6), pp. 3722 - 3736. https://doi.org/10.9756/sijcnce/v4i1/04010030101

[15] Chand, K., K. D. Singh and S. Kumar (2012). Hall effect on radiating and chemically reacting MHD oscillatory flow in a rotating porous vertical channel in slip flow regime. Pelagia Research Library Advances in Applied Science Research Vol. 3(4), pp. 2424 - 2437. 
M. Nikodijevic, Z. Stamenkovic, J. Petrovic, M. Kocic
Unsteady Fluid Flow and Heat Transfer Through a Porous Medium in a Horizontal Channel with an Inclined Magnetic Field

[16] Choudhury, R. and S. K. Das (2014). Visco-Elastic MHD Free Convective Flow through Porous Media in Presence of Radiation and Chemical Reaction with Heat and Mass Transfer. Journal of Applied Fluid Mechanics Vol. 7(4), pp. 603 - 609. https://doi.org/10.36884/jafm.7.04.20307

[17] Uwanta, I. J. and H. Usman (2014). On the Influence of Soret and Dufour Effects on MHD Free Convective Heat and Mass Transfer Flow over a Vertical Channel with Constant Suction and Viscous Dissipation. International Scholarly Research Notices Vol. 2014(639159), 11 pages. https://doi.org/10.1155/2014/639159

[18] Barik, R. N., G.C. Dash and M. Kar (2014). Unsteady free convective MHD flow and mass transfer through porous medium in a rotating system with fluctuating heat source/sink and chemical reaction. Journal of Applied Analysis and Computation Vol. 4(3), pp. 231 - 244. https://doi.org/10.11948/2014011

[19] Prakash, O. M., O.D. Makinde, D. Kumar and Y. K. Dwivedi (2015). Heat transfer to MHD oscillatory dusty fluid flow in a channel filled with a porous medium. Sadhana Vol. 40(4), pp.1273 - 1282. https://doi.org/10.1007/s12046-015-0371-9

[20] Kuppala, S. R. and V. G. Reddy (2015). Unsteady MHD Convective Heat and Mass Transfer of a Casson Fluid Past a Semi-infinite Vertical Permeable Moving Plate with Heat Source/Sink. Chemical and Process Engineering Research Vol.39, pp. 5 - 12. https://doi.org/10.1016/j.proeng.2015.11.352

[21] Kumar, S. R (2016). MHD Peristaltic Transportation of a Conducting Blood Flow with Porous Medium through Inclined Coaxial Vertical Channel. International Journal of Bio - Science and Bio - Technology Vol. 8(1), pp.11 - 26. https://doi.org/10.14257/ijbsbt.2016.8.1.02

[22] Misra, J. C. and S. D. Adhikary (2016). MHD oscillatory channel flow, heat and mass transfer in a physiological fluid in presence of chemical reaction. Elsevier, Alexandria Engineering Journal Vol. 55, pp. 287 - 297. https://doi.org/10.1016/j.aej.2015.10.005

[23] Falade, J. A., J. C. Ukaeglu, A. C. Egere and S. O. Adesanya (2017). MHD oscillatory flow through a porous channel saturated with porous medium. Alexandria Engineering Journal Vol. 56, pp. 147 - 152. https://doi.org/10.1016/j.aej.2016.09.016

[24] Krishna, M. V., G. S. Reddy and A. J. Chamkha (2018). Hall effects on unsteady MHD oscillatory free convective flow of second grade fluid through porous medium between two vertical plates. Physics of fluids Vol. 30(023106). https://doi.org/10.1063/1.5010863

[25] Scheel, J. D. and J. Schumacher (2016). Global and local statistics in turbulent convection at low Prandtl numbers. Journal of Fluid Mechanics Vol. 802, pp. 147 - 173. https://doi.org/10.1017/jfm.2016.457

Submitted: $\quad 30.01 .2020$

Accepted: $\quad 30.5 .2020$
Milica Nikodijevic*

milica.nikodijevic@znrfak.ni.ac.rs

Faculty of Occupational Safety, University of Nis, Čarnojevića 10a, 18000 Niš, Serbia Zivojin Stamenkovic zivojin.stamenkovic@masfak.ni.ac.rs Jelena Petrovic jelena.nikodijevic.petrovic@masfak.ni.ac.rs Milos Kocic milos.kocic@masfak.ni.ac.rs Faculty of Mechanical Engineering, University of Nis, Aleksandra Medvedeva 14, 18000 Nis, Serbia 